\title{
Numerical Solution of Two Dimensional Stagnation Flows of Newtonian Fluid Towards a Shrinking Sheet
}

\author{
Mohammad Shafique ${ }^{1, *}$, Fatima Abbas ${ }^{1}$, Atif Nazir ${ }^{2}$ \\ ${ }^{1}$ Department of Mathematics, Gomal University, Pakistan \\ ${ }^{2}$ Mathematics Group, Yanbu Industrial College, Saudi Arabia
}

Copyright (C) 2015 Horizon Research Publishing All rights reserved.

\begin{abstract}
The two dimensional stagnation flows towards a shrinking sheet of Newtonian fluids has been solved numerically by using SOR Iterative Procedure. The similarity transformations have been used to reduce the highly nonlinear partial differential equations to ordinary differential equations. The results have been calculated on three different grid sizes to check the accuracy of the results. The problem relates to the flows towards a shrinking sheet when $\alpha<0$ and if $\alpha>0$ the flows towards a stretching sheet. The numerical results for Newtonian fluids are found in good agreement with those obtained previously.
\end{abstract}

Keywords Newtonian fluids, Shrinking Sheet and SOR Iterative Procedure

AMS Subject Classification: 76D99. 76M20, 65N22

\section{Introduction}

The two dimensional fluid flow near a stagnation point is among the fundamental problems in fluid mechanics. The study of stagnation point flow has been extended in numerous ways including MHD flow, heat transfer, and porous medium and stretching surfaces. In recent years, the stagnation flow problem is being investigated for shrinking boundaries. Fang and Zhang [1] considered MHD flow over a shrinking sheet and obtained closed form exact solution for the problem. The MHD boundary layer flow of fluid over a shrinking sheet has been studied by Hayat et al [2] and Fang [3]. Nadeem et al [4] and Ara et al [5] have been investigated MHD boundary layer flow of fluid over an exponentially permeable shrinking sheet. The steady boundary layer flow and steady two-dimensional flow of a nanofluid past a nonlinearly permeable stretching/ shrinking sheet is numerically studied by Zaimi et al [6, 7]. Sajid and Hayat [8] applied homotopy analysis method for MHD viscous flow due to a shrinking sheet. The problem of [8] is studied by Noor et al. [9] by using simple non-perturbative method. Wang [10] studied the stagnation flow towards a shrinking sheet.

In this paper, the numerical solutions of stagnation flow towards a shrinking sheet for Newtonian fluid have been discussed. In order to find the numerical solution of the problem, the Navier Stokes equations are reduced to ordinary differential equations by using similarity transformations [10]. The resulting equations are solved numerically by using SOR method and Simpson's (1/3) rule, for various values of the parameter $\alpha$ and the Prandtle number Pr. When $\alpha>0$, the problem relates to the stagnation flow towards a stretching sheet. When $\alpha=0$, it becomes Hiemenz [11] flow towards a solid plate. The problem relates to the flow towards a shrinking sheet when $\alpha<0$.

\section{Mathematical Analysis}

The continuity equation and the Navier-Stokes equations for incompressible fluid in the absence of body forces are given by

$$
\begin{gathered}
\nabla \cdot \underline{V}=0, \\
\rho(\underline{V} \cdot \nabla) \underline{V}=-\nabla \pi+\mu \nabla^{2} \underline{V}, \\
\rho C_{p}(\underline{V} \cdot \nabla) T=K \nabla^{2} T .
\end{gathered}
$$

where $\rho$ is the density, $V$ is the velocity vector, $\pi$ is the pressure and $\mu$ viscosity coefficient. The fluid temperature is $T$ where as $\Phi, \mathrm{C}_{\mathrm{p}}$ and $K$ denote the dissipation function (in this case the dissipation function is neglected), specific heat and heat conductivity respectively.

The flow is in the frame of Cartesian coordinates. The velocity vector is represented by $\underline{V}=V(u, 0, w)$. The two dimensional potential stagnation flow at infinity is given by $u=a x, w=-a z$ where $a$ is the strength of the stagnation flow. The velocity components, on the stretching surface are $u=b(x+c), w=0$, where $b$ is stretching rate and $b<0$ indicates the shrinking of the surface. The stretching origin is located at $-c$.

Under these assumptions, the set of equations (1) to (3) become:

$$
\frac{\partial u}{\partial x}+\frac{\partial w}{\partial z}=0,
$$




$$
\begin{gathered}
\mu\left(\frac{\partial^{2} u}{\partial x^{2}}+\frac{\partial^{2} u}{\partial z^{2}}\right)-\frac{\partial \pi}{\partial x}=\rho\left(u \frac{\partial u}{\partial z}+w \frac{\partial u}{\partial z}\right) \\
\mu\left(\frac{\partial^{2} w}{\partial x^{2}}+\frac{\partial^{2} w}{\partial z^{2}}\right)-\frac{\partial \pi}{\partial z}=\rho\left(u \frac{\partial w}{\partial z}+w \frac{\partial w}{\partial z}\right) \\
\rho C_{p}\left(u \frac{\partial T}{\partial x}+w \frac{\partial T}{\partial z}\right)=K\left(\frac{\partial^{2} T}{\partial x^{2}}+\frac{\partial^{2} T}{\partial z^{2}}\right) .
\end{gathered}
$$

The following similarity transformations of [10] are used to convert the governing partial differential equations into ordinary differential form:

$$
\begin{aligned}
& u=a x f^{\prime}(\eta)+b c g(\eta), \\
& w=-\sqrt{v a} f(\eta) \text { and } \theta(\eta)=\frac{T-T_{\infty}}{T_{0}-T_{w}},
\end{aligned}
$$

where $\eta=\sqrt{\frac{a}{v}} z$ is a dimensionless variable. The functions $f, f^{\prime}, g$ and $\theta$ are the similarity, velocity, non-alignment and temperature profiles respectively.

The equation of continuity (4) is readily satisfied. From equation (6), the pressure $\pi$ is obtained as:

$$
\pi=\pi_{0}+\rho a^{2} \frac{x^{2}}{2}-\rho \frac{w^{2}}{2}+\rho v \frac{\partial w}{\partial z} .
$$

The equations (5) and (7) by using (8) yield a set of non linear ordinary differential equations:

$$
\begin{gathered}
f^{\prime \prime \prime}+1=f^{\prime 2}-f^{\prime \prime} f, \\
g^{\prime \prime}=g f^{\prime}-g^{\prime} f, \\
\theta^{\prime \prime}+\operatorname{Pr} f \theta^{\prime}=0 .
\end{gathered}
$$

The boundary conditions are

$$
\begin{cases}f=0, f^{\prime}=\alpha, g=1, \theta=1 & \text { at } \eta=0 \\ f^{\prime} \rightarrow 1, g \rightarrow 0, \theta \rightarrow 0 & \text { as } \eta \rightarrow \infty\end{cases}
$$

Where $\alpha=\frac{b}{a}, \operatorname{Pr}=\frac{\rho v C_{p}}{K}$ and $v=\frac{\mu}{\rho}$ is the coefficient of kinematics viscosity. The prime denotes the differentiation with respect to $\eta$.

\section{Finite Difference Equations}

To obtain numerical solution of the equations (10) and (11), we let

$$
P=f^{\prime}
$$

Then equations (10) and (11) become

$$
\begin{aligned}
P^{\prime \prime}+1 & =P^{2}-f P^{\prime}, \\
g^{\prime \prime} & =g P-g^{\prime} f,
\end{aligned}
$$

with boundary conditions

$$
\begin{cases}f=0, P=\alpha, g=1, \theta=1 & \eta=0, \\ P=1, g=0, \theta=0 & \eta \rightarrow \infty .\end{cases}
$$

The equations (15), (16) and (12) by using central difference approximations at a typical point $\eta=\eta_{n}$ of the interval $[0, \infty)$ and the resulting finite difference equations are obtained below

$$
\begin{gathered}
\left(4+2 h^{2} P_{n}\right) P_{n}=2 h^{2}+\left(2+h f_{n}\right) P_{n+1}+\left(2-h f_{n}\right) P_{n-1}, \\
\left(4+2 h^{2} P_{n}\right) g_{n}=\left(2+h f_{n}\right) g_{n+1}+\left(2-h f_{n}\right) g_{n-1}, \\
2\left(\theta_{n+1}+\theta_{n-1}\right)+\operatorname{Pr} f_{n} h\left(\theta_{n+1}-\theta_{n-1}\right)=4 \theta_{n},
\end{gathered}
$$

where $h$ denotes the grid size and the symbols used denote $f_{n}=f\left(\eta_{n}\right), P_{n}=P\left(\eta_{n}\right), g_{n}=g\left(\eta_{n}\right)$ and $\theta_{n}=\theta\left(\eta_{n}\right)$. For computational purposes, we replace the interval $[0, \infty)$ by $[0, \beta)$ where $\beta$ is sufficiently large.

\section{Computational Procedure}

We now solve numerically the first order ordinary differential equation (14) and the system of finite difference equations (18) to (20) at each interior grid point of the interval. The equation (14) is integrated by the Simpson's $(1 / 3)$ rule, whereas the set of equations (18) to (20) are solved by using SOR iterative procedure subject to the appropriate conditions.

The order of the sequence of iterations is as follows:

1. The equations (18) to (20) are solved to calculate the value of $P, g$ and $\theta$ subject to the boundary conditions

$$
\begin{aligned}
& P=\alpha, \quad g=1, \quad \theta=1, \quad \text { when } \quad \eta=0, \\
& P=1, \quad g=0, \quad \theta=0, \quad \text { when } \quad \eta \rightarrow \infty .
\end{aligned}
$$

1. The computed solution of $P$ is then employed into the equation (14) for the calculation of $f$ with the condition:

$$
f=0 \quad \text { when } \quad \eta=0
$$

1. In order to accelerate the speed of convergence of the SOR method, the optimum value of the relaxation parameter $\omega_{\text {opt }}$ is estimated between 1 and 2 . The optimum value of the relaxation parameter for the problem under consideration is 1.5 .

2. The above procedure is repeated until convergence is obtained according to the criterion $\max \left|U^{n+1}-U^{n}\right|<10^{-6}$ where $n$ denotes the number of iterations and $U$ stands for each of the functional value.

For higher order accuracy, the above steps 1 to 4 are repeated for step sizes $\frac{h}{2}$ and $\frac{h}{4}$. 


\section{Discussion on Numerical Results}

The calculations have been carried out for the values of parameter $\alpha$ in the range $-1.2475 \leq \alpha \leq 10$. The accuracy of the results is checked by comparing them on different grid sizes namely $\mathrm{h}=0.01,0.005,0.0025$. The results are presented in the Table 1 to Table 5, for the functions $f, f^{\prime}, g$ and $\theta$ for each of grid size. The results compare quite well to validate our computational procedure. The present results for $f^{\prime \prime}(0)$ and $g^{\prime}(0)$ have been compared with the previous results by Wang [10]. This comparison of the results is shown in the Table 6.

Table 1. The Numerical solutions of $f, f^{\prime}, g$ and $\theta$ for $\alpha=-1.2475$

\begin{tabular}{|c|c|c|c|c|c|}
\hline $\mathrm{h}$ & $\eta$ & $f$ & $f^{\prime}$ & $\mathrm{g}$ & $\theta$ \\
\hline \multirow{5}{*}{0.010} & 0.000 & 0.000000 & -1.247500 & 1.000000 & 1.000000 \\
\cline { 2 - 6 } & 1.000 & -0.873815 & -0.463615 & 1.352902 & 0.922083 \\
\cline { 2 - 6 } & 2.000 & -0.938034 & 0.299056 & 1.011058 & 0.784882 \\
\cline { 2 - 6 } & 3.000 & -0.376772 & 0.768402 & 0.485995 & 0.535224 \\
\cline { 2 - 6 } & 4.000 & 0.502791 & 0.954425 & 0.130963 & 0.222099 \\
\cline { 2 - 6 } & 5.000 & 1.485921 & 1.000000 & 0.000000 & 0.000000 \\
\hline \multirow{5}{*}{0.005} & 0.000 & 0.000000 & -1.247500 & 1.000000 & 1.000000 \\
\cline { 2 - 6 } & 1.000 & -0.879391 & -0.473710 & 1.348155 & 0.895986 \\
\cline { 2 - 6 } & 2.000 & -0.955276 & 0.287303 & 1.006417 & 0.736884 \\
\cline { 2 - 6 } & 3.000 & -0.403708 & 0.761322 & 0.484015 & 0.484802 \\
\cline { 2 - 6 } & 4.000 & 0.471404 & 0.952222 & 0.131038 & 0.196441 \\
\cline { 2 - 6 } & 5.000 & 1.453628 & 1.000000 & 0.000000 & 0.000000 \\
\hline \multirow{5}{*}{0.0025} & 0.000 & 0.000000 & -1.247500 & 1.000000 & 1.000000 \\
\cline { 2 - 6 } & 1.000 & -0.879424 & -0.473734 & 1.006821 & 0.736738 \\
\cline { 2 - 6 } & 2.000 & -0.955243 & 0.287451 & 0.132263 & 0.196995 \\
\cline { 2 - 6 } & 3.000 & -0.403563 & 0.761347 & 0.000003 & 0.000005 \\
\cline { 2 - 6 } & 4.000 & 0.471552 & 0.952210 & 0.000000 & 0.000000 \\
\cline { 2 - 6 } & 5.000 & 1.453769 & 1.000000 & 0.000000 & 0.000000 \\
\hline
\end{tabular}

Table 2. The Numerical solutions of $f, f^{\prime}, g$ and $\theta$ for $\alpha=-1.0$

\begin{tabular}{|c|c|c|c|c|c|}
\hline$h$ & $\eta$ & $f$ & $f^{\prime}$ & $g$ & $\theta$ \\
\hline \multirow{6}{*}{0.010} & 0.000 & 0.000000 & -1.000000 & 1.000000 & 1.000000 \\
\hline & 1.000 & -0.377963 & 0.171266 & 0.687185 & 0.751338 \\
\hline & 2.000 & 0.147423 & 0.784151 & 0.261551 & 0.444435 \\
\hline & 3.000 & 1.046319 & 0.968087 & 0.053732 & 0.171482 \\
\hline & 4.000 & 2.034327 & 0.997696 & 0.005192 & 0.035421 \\
\hline & 5.000 & 3.033712 & 1.000000 & 0.000000 & 0.000000 \\
\hline \multirow{6}{*}{0.005} & 0.000 & 0.000000 & -1.000000 & 1.000000 & 1.000000 \\
\hline & 1.000 & -0.377917 & 0.171507 & 0.312491 & 0.453647 \\
\hline & 2.000 & 0.147851 & 0.784538 & 0.019946 & 0.059923 \\
\hline & 3.000 & 1.046918 & 0.968079 & 0.000304 & 0.001450 \\
\hline & 4.000 & 2.034912 & 0.997628 & 0.000002 & 0.000012 \\
\hline & 5.000 & 3.034207 & 1.000000 & 0.000000 & 0.000000 \\
\hline \multirow{6}{*}{0.0025} & 0.000 & 0.000000 & -1.000000 & 1.000000 & 1.000000 \\
\hline & 1.000 & -0.377914 & 0.171579 & 0.021163 & 0.061557 \\
\hline & 2.000 & 0.147970 & 0.784652 & 0.000002 & 0.000015 \\
\hline & 3.000 & 1.047083 & 0.968072 & 0.000000 & 0.000000 \\
\hline & 4.000 & 2.035067 & 0.997630 & 0.000000 & 0.000000 \\
\hline & 5.000 & 3.034366 & 1.000000 & 0.000000 & 0.000000 \\
\hline
\end{tabular}


Table 3. The Numerical solutions of $f, f^{\prime}, g$ and $\theta$ for $\alpha=0.0$, same as of Hiemenz [11]

\begin{tabular}{|c|c|c|c|c|c|}
\hline $\mathrm{h}$ & $\eta$ & $f$ & $f^{\prime}$ & $\mathrm{g}$ & $\theta$ \\
\hline \multirow{5}{*}{0.010} & 0.000 & 0.000000 & 0.000000 & 1.000000 & 1.000000 \\
\cline { 2 - 6 } & 1.000 & 0.459239 & 0.777891 & 0.322968 & 0.519335 \\
\cline { 2 - 6 } & 2.000 & 1.362030 & 0.973288 & 0.053424 & 0.173112 \\
\cline { 2 - 6 } & 3.000 & 2.352734 & 0.998610 & 0.004120 & 0.032825 \\
\cline { 2 - 6 } & 4.000 & 3.352518 & 1.000164 & 0.000135 & 0.003210 \\
\cline { 2 - 6 } & 5.000 & 4.352617 & 1.000000 & 0.000000 & 0.000000 \\
\cline { 2 - 6 } & 0.000 & 0.000000 & 0.000000 & 1.000000 & 1.000000 \\
\cline { 2 - 6 } & 1.000 & 0.459337 & 0.778030 & 0.171640 & 0.268332 \\
\cline { 2 - 6 } & 2.000 & 1.362234 & 0.973350 & 0.009834 & 0.027797 \\
\cline { 2 - 6 } & 3.000 & 2.352902 & 0.998360 & 0.000203 & 0.001126 \\
\cline { 2 - 6 } & 4.000 & 3.352365 & 0.999859 & 0.000002 & 0.000020 \\
\cline { 2 - 6 } & 5.000 & 4.352293 & 1.000000 & 0.000000 & 0.000000 \\
\hline \multirow{5}{*}{0.005} & 0.000 & 0.000000 & 0.000000 & 1.000000 & 1.000000 \\
\cline { 2 - 6 } & 1.000 & 0.459373 & 0.778100 & 0.010421 & 0.028715 \\
\cline { 2 - 6 } & 2.000 & 1.362321 & 0.973384 & 0.000002 & 0.000023 \\
\cline { 2 - 6 } & 3.000 & 2.352996 & 0.998363 & 0.000000 & 0.000000 \\
\cline { 2 - 6 } & 4.000 & 3.352462 & 0.999864 & 0.000000 & 0.000000 \\
\cline { 2 - 6 } & 5.000 & 4.352391 & 1.000000 & 0.000000 & 0.000000 \\
\hline \multirow{6}{*}{0.0025} & & & & & \\
\hline
\end{tabular}

Table 4. The Numerical solutions of $f, f^{\prime}, g$ and $\theta$ for $\alpha=5.0$

\begin{tabular}{|c|c|c|c|c|c|}
\hline $\mathrm{h}$ & $\eta$ & $f$ & $f^{\prime}$ & $\mathrm{g}$ & $\theta$ \\
\hline \multirow{6}{*}{0.010} & 0.000 & 0.000000 & 5.000000 & 1.000000 & 1.000000 \\
\hline & 1.000 & 2.370658 & 1.217793 & 0.069796 & 0.200439 \\
\hline & 2.000 & 3.431455 & 1.005381 & 0.002158 & 0.019628 \\
\hline & 3.000 & 4.432723 & 1.000166 & 0.000027 & 0.001002 \\
\hline & 4.000 & 5.432887 & 1.000145 & 0.000000 & 0.000026 \\
\hline & 5.000 & 6.432961 & 1.000000 & 0.000000 & 0.000000 \\
\hline \multirow{6}{*}{0.005} & 0.000 & 0.000000 & 5.000000 & 1.000000 & 1.000000 \\
\hline & 1.000 & 2.370430 & 1.217636 & 0.034929 & 0.080309 \\
\hline & 2.000 & 3.431183 & 1.005423 & 0.000269 & 0.001563 \\
\hline & 3.000 & 4.432415 & 1.000017 & 0.000001 & 0.000010 \\
\hline & 4.000 & 5.432386 & 0.999942 & 0.000000 & 0.000000 \\
\hline & 5.000 & 6.432336 & 1.000000 & 0.000000 & 0.000000 \\
\hline \multirow{6}{*}{0.0025} & 0.000 & 0.000000 & 5.000000 & 1.000000 & 1.000000 \\
\hline & 1.000 & 2.370227 & 1.217539 & 0.000914 & 0.003416 \\
\hline & 2.000 & 3.430947 & 1.005529 & 0.000000 & 0.000000 \\
\hline & 3.000 & 4.432255 & 1.000096 & 0.000000 & 0.000000 \\
\hline & 4.000 & 5.432298 & 0.999991 & 0.000000 & 0.000000 \\
\hline & 5.000 & 6.432321 & 1.000000 & 0.000000 & 0.000000 \\
\hline
\end{tabular}


Table 5. The Numerical solutions of $f, f^{\prime}, g$ and $\theta$ for $\alpha=10.0$

\begin{tabular}{|c|c|c|c|c|c|}
\hline $\mathrm{h}$ & $\eta$ & $f$ & $f^{\prime}$ & $\mathrm{g}$ & $\theta$ \\
\hline \multirow{6}{*}{0.010} & 0.000 & 0.000000 & 10.000000 & 1.000000 & 1.000000 \\
\hline & 1.000 & 3.456570 & 1.205824 & 0.028125 & 0.108641 \\
\hline & 2.000 & 4.503183 & 1.002047 & 0.000332 & 0.005310 \\
\hline & 3.000 & 5.503655 & 1.000163 & 0.000002 & 0.000133 \\
\hline & 4.000 & 6.503815 & 1.000130 & 0.000000 & 0.000002 \\
\hline & 5.000 & 7.503879 & 1.000000 & 0.000000 & 0.000000 \\
\hline \multirow{6}{*}{0.005} & 0.000 & 0.000000 & 10.000000 & 1.000000 & 1.000000 \\
\hline & 1.000 & 3.456435 & 1.205746 & 0.019623 & 0.055949 \\
\hline & 2.000 & 4.503034 & 1.002028 & 0.000098 & 0.000783 \\
\hline & 3.000 & 5.503368 & 0.999968 & 0.000000 & 0.000004 \\
\hline & 4.000 & 6.503291 & 0.999919 & 0.000000 & 0.000000 \\
\hline & 5.000 & 7.503234 & 1.000000 & 0.000000 & 0.000000 \\
\hline \multirow{6}{*}{0.0025} & 0.000 & 0.000000 & 10.000000 & 1.000000 & 1.000000 \\
\hline & 1.000 & 3.456014 & 1.205641 & 0.000665 & 0.002760 \\
\hline & 2.000 & 4.502621 & 1.002135 & 0.000000 & 0.000000 \\
\hline & 3.000 & 5.503085 & 1.000080 & 0.000000 & 0.000000 \\
\hline & 4.000 & 6.503112 & 0.999994 & 0.000000 & 0.000000 \\
\hline & 5.000 & 7.503150 & 1.000000 & 0.000000 & 0.000000 \\
\hline
\end{tabular}

Table 6. The comparison of present results for $f^{\prime \prime}(0)$ and $g^{\prime}(0)$ with the previous results

\begin{tabular}{|c|c|c|c|c|}
\hline \multirow{2}{*}{$\alpha$} & \multicolumn{2}{|c|}{$f^{\prime \prime}(0)$} & \multicolumn{2}{c|}{$g^{\prime}(0)$} \\
\cline { 2 - 5 } & Present Results & Results of Wang[89] & Present Results & Results of Wang[89] \\
\hline 0.0 & 1.230355 & 1.232588 & -0.8110881 & -0.811301 \\
\hline 0.1 & 1.146591 & 1.14656 & -0.8629799 & -0.86345 \\
\hline 0.2 & 1.051265 & 1.05113 & -0.9122908 & -0.91330 \\
\hline 0.5 & 0.713372 & 0.71330 & -1.0489881 & -1.05239 \\
\hline 1.0 & -0.00007 & 0.00000 & -1.2480258 & -1.25331 \\
\hline 2.0 & -1.8854618 & -1.88731 & -1.5795648 & -1.58957 \\
\hline 5.0 & -10.239030 & -10.26475 & -2.313268 & -2.33810 \\
\hline 8.0 & -21.610450 & ---- & -2.865630 & --- \\
\hline 10.0 & -30.164527 & ---- & -3.179997 & --- \\
\hline-0.05 & 1.271822 & ---- & -0.7838547 & -- \\
\hline-0.15 & 1.342702 & ---- & -0.7285535 & -0.66857 \\
\hline-0.25 & 1.402199 & 1.40224 & -0.6691158 & -0.50145 \\
\hline-0.5 & 1.495576 & 1.49567 & -0.5033791 & -0.29376 \\
\hline-0.75 & 1.488662 & 1.48930 & -0.2966404 & 0.0 \\
\hline-1.0 & 1.3282775 & 1.32882 & -0.004076 & 0.297995 \\
\hline-1.15 & 1.0814666 & 1.08223 & 0.2933860 & 0.99904 \\
\hline-1.2465 & 0.6310463 & 0.55430 & 0.8562684 & --- \\
\hline-1.2475 & 0.6090641 & --- & 0.8745313 & \\
\hline
\end{tabular}


Graphically, the results for the function $f$ are shown in Figure 1 and Figure 2. It is noticed that the function $f$ increases with the increasing values of $\alpha$ positive. When $\alpha=0, f$ behaves like the Hiemenz [11] flow towards a solid plate. For negative values of $\alpha$, the function $f$ is initially negative. The situation depicts the regions of reverse cellular flow. The effect of $\alpha$ on the universal function $g$ is shown in Figure 3.The effect is smaller in case of stretching sheet but larger for shrinking sheet. The graph of $f^{\prime \prime}(0)$ and $g^{\prime}(0)$ have been plotted respectively in Figure 4 and Figure 5. The non-alignment function $g$ has no effect on heat transfer. For increasing values of the shrinking parameter $\alpha$, the boundary layer thickness increases and hence the heat transfer rate decreases as depicted in Figure 6.

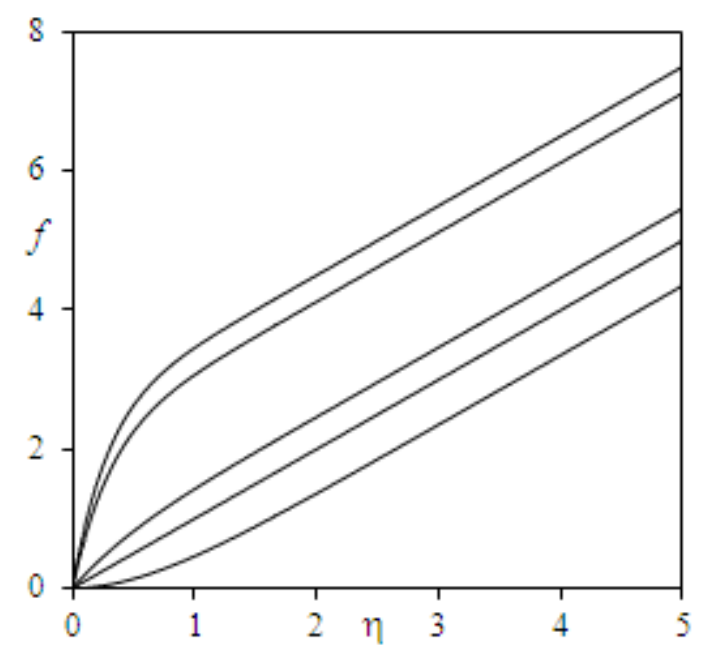

Figure 1. Graph of $f$ for different values of $\alpha=0,1,2,5$ and 10 from bottom

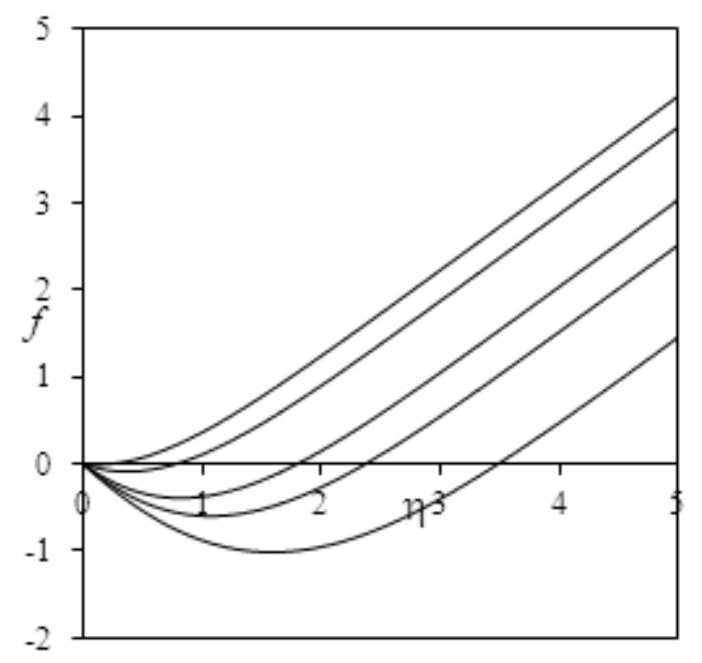

Figure 2. Graph of $f$ for different values of $\alpha=-0.5,-0.75,-1,-1.15$ and -1.2475 from top

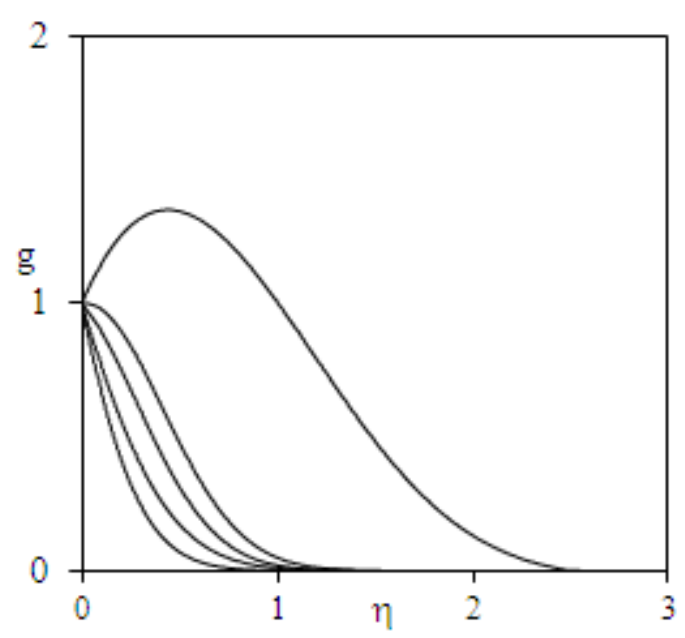

Figure 3. Graph of $g$ for different values of $\alpha=1,0,-1,-1.15$ and -1.2475 from bottom.

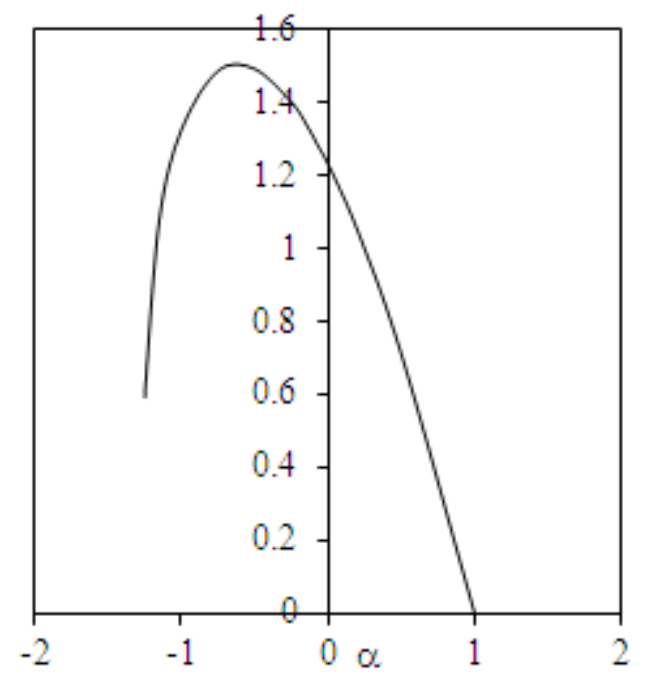

Figure 4. Graph of the skin friction coefficient $f^{\prime \prime}(0)$.

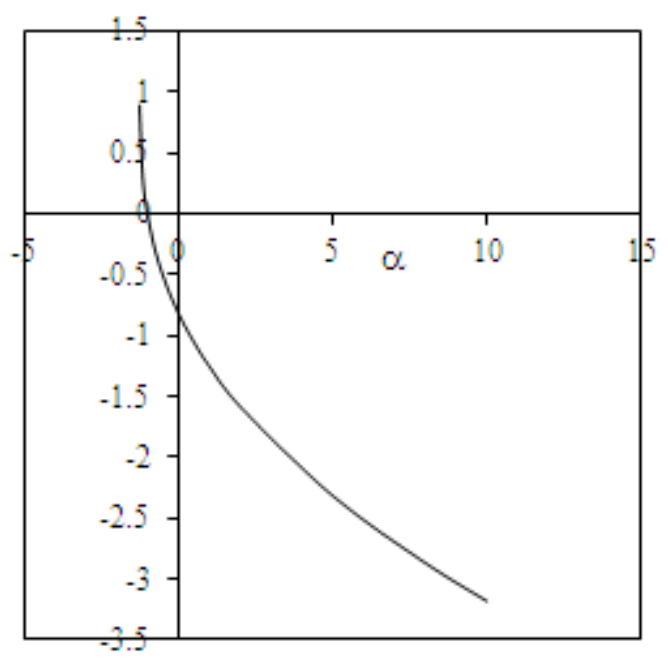

Figure 5: Graph of the gradient of non alignment function $g$ i.e. $g^{\prime}(0)$ 


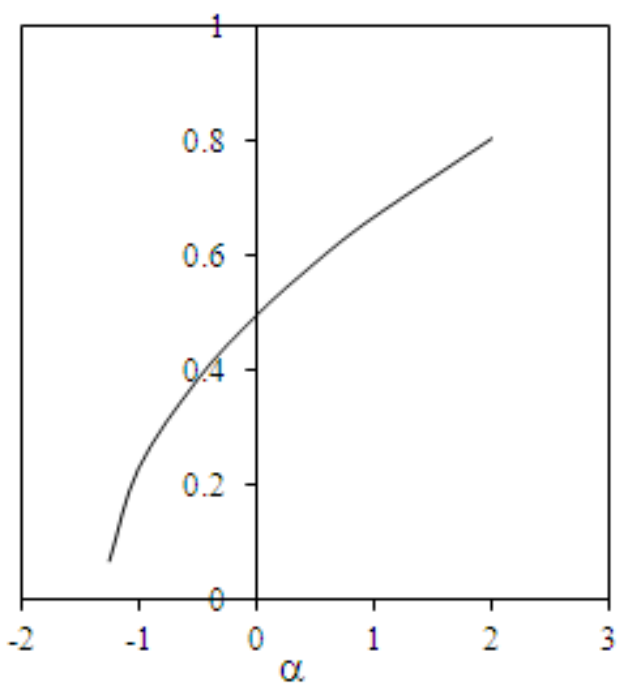

Figure 6. Graph of $-\theta^{\prime}(0)$ when $\operatorname{Pr}=0.7$

\section{Conclusions}

The effects of parameter $\alpha$ in the extended range $-1.2475 \leq \alpha \leq 10$ are observed on the similarity, velocity, non-alignment and temperature profiles. Consequently, the function $f$ increases with the increasing values of $\alpha$ positive. When $\alpha=0, f$ behaves like the Hiemenz flow towards a solid plate. The non-alignment function $g$ has no effect on heat transfer. For increasing values of the shrinking parameter $\alpha$, the boundary layer thickness increases and hence the heat transfer rate decreases. Thus the numerical results in this work are found in good agreement with those obtained previously.

\section{REFERENCES}

[1] Fang, T. and Zhang, Ji. (2009), Closed- Form exact solutins of MHD viscous flow over a shrinking sheet, Commun Nonlinear Sci Numer Sumulat, 14, 2853 -2857.

[2] T. Hayat, Z. Abbas and M. Sajid, (2007), On the Analytic Solution of MHD Flow of a Second Grade Fluid Over a Shrinking Sheet, .J. Appl. Mech. 74(6), 1165-1171

[3] Tiegang Fang, (2008), Boundary layer flow over a shrinking sheet with power-law velocity, International Journal of Heat and Mass Transfer, Volume 51, Issues 25-26, 5838-5843.

[4] S. Nadeem, Rizwan Ul Haq, C. Lee, (2012), MHD flow of a Casson fluid over an exponentially shrinking sheet,Scientia Iranica B 19 (6), 1550-1553.

[5] Asmat Ara, Najeeb Alam Khan, Hassam Khan, Faqiha Sultan, (2014), Radiation effect on boundary layer flow of an EyringPowell fluid over an exponentially shrinking sheet, Ain Shams Engineering Journal, Volume 5, Issue 4, 1337-1342.

[6] Khairy Zaimi, Anuar Ishak \& Ioan Pop, (2014), Boundary layer flow and heat transfer over a nonlinearly permeable stretching/shrinking sheet in a nanofluid, Scientific ReportsVolume: 4, Article number:4404 DOI: doi:10.1038/ srep04404.

[7] Khairy Zaimi,, Anuar Ishak, Ioan Pop , (2014), Flow Past a Permeable Stretching/Shrinking Sheet in a Nanofluid Using Two-Phase Model, PLoS ONE 9(11): e111743.

[8] Sajid, M. and Hayat, T. (2009), The application of homotopy analysis method for MHD viscous flow due to a shrinking sheet, Chaors Soliton Fractals, 39, 1317-1323.

[9] Noor, N. F. M., Kechil, S. A. and Hashim, I. (2010), Simple non-perturbative solution for MHD viscous flow due to a shrinking sheet, Commun Nonlinear Sci Numer Sim Ilat 15, 144-148.

[10] Wang. C.Y. (2008), Stagnation flow towards a shrinking sheet, International journal of Non-Linear Mechanics, 43, 377 -382 .

[11] Hiemenz, K. (1911), Die Grenzschicht an einem in den gleichformingen flussigkeitsstrom eingetauchten garden Kreiszylinder, Dinglers Polytech. J., 326, 321-324.

[12] C. F. Gerald, "Applied Numerical Analysis," AddisonWesley Publication, New York, 1989. 